Article

\title{
The Effect of Glancing Angle Deposition Conditions on the Morphology of a Silver Nanohelix Array
}

\author{
Yi-Jun Jen *, Yueh Weng Lin, Yu-Jie Huang and Teh-Li Chan \\ Department of Electro-Optical Engineering, National Taipei University of Technology, No. 1, Sec. 3, \\ Chung-Hsiao E. Rd., Taipei 106, Taiwan; jeter513@hotmail.com (Y.W.L.); eternal810219@gmail.com (Y.-J.H.); \\ derek61104@gmail.com (T.-L.C.) \\ * Correspondence: jyjun@ntut.edu.tw; Tel.: +886-2-2771-2171 (ext. 4626)
}

Received: 13 July 2017; Accepted: 31 August 2017; Published: 4 September 2017

\begin{abstract}
Silver nanohelices were grown on smooth substrates using glancing angle deposition and substrate cooling. Various nanohelix arrays were deposited under different deposition conditions-different deposition rates, substrate spin rates, deposition angles, and substrate temperatures. The effect of deposition conditions on the morphology of each nanohelix array in terms of pitch angle, pitch length, wire diameter, and radius of curvature was investigated. The dependence of circular dichroism on the size of the nanohelix arrays was also measured and demonstrated.
\end{abstract}

Keywords: glancing angle deposition; nanohelix; g-factor

\section{Introduction}

The optical properties of plasmonic helix arrays have been demonstrated to depend strongly on circular polarization states. In 2008, Martin Wegener et al. [1] prepared regularly distributed gold nanohelices with a pitch length of $1.75 \mu \mathrm{m}$ and a radius of curvatures of $0.75 \mu \mathrm{m}$ using a laser writing technique for use as a broadband polarizer. They systematically studied the dependence of the chiral optical properties on helix pitch, the radius of curvature, the two-dimensional lattice constant, wire diameter, the number of helix pitches, and the angle of incidence using a numerical method [2]. To shift the wavelengths corresponding to chiral plasmonic resonance from infrared toward the visible regime, the size of the helices had to be reduced by one order of magnitude.

Owing to the development of coatings, nanohelices with feature sizes of less than $100 \mathrm{~nm}$ can be produced using a cheap and easy method called glancing angle deposition (GLAD). The GLAD technique is a unique technique for wafer-scale deposition via a one-step process [3,4]. In 2013, Mark et al. [5] employed an extremely low substrate temperature of approximately $-140{ }^{\circ} \mathrm{C}$ to obliquely deposit gold nanohelices with pitch lengths of only $34 \mathrm{~nm}$ on a seeded surface. The low substrate temperature was required to reduce the diffusion energy of deposited atoms, and the seeds on the surface were arranged to offer a shadowing effect [6]. However, expensive lithography is required to pattern the seeded surface of the substrate [7]. Recent work has revealed that pure metal helix arrays can be obliquely deposited on smooth substrates via self-shadowing [8-11]. Rather than using liquid nitrogen to cool the substrate to a temperature of around $-140{ }^{\circ} \mathrm{C}$, metal helix arrays can be grown on a substrate at a temperature of $10{ }^{\circ} \mathrm{C}$ using ice water for substrate cooling, as has been proposed in recent works [12].

It has been demonstrated that metal nanohelices have played an important role in the pharmaceutical industry [13]. The optical chirality of a metal nanohelix is enhanced due to the localized plasmon excitation. When chiral molecules are in the vicinity of plasmonic chiral particles, the chiroptical interaction will be significantly changed to detect the molecules. For example, the chiroptical activity of silver nanospirals grafted with achiral alkyl ligands is weakened due to the effective medium screening effect [14]. 
In conventional coating, the columnar morphology of a deposited thin film depends mainly on the temperature of the substrate $\left(T_{\mathrm{s}}\right)$ and the melting temperature of the coating material $\left(T_{\mathrm{m}}\right)$. A zone model was constructed and applied to estimate the nanostructure of a thin film from the two temperatures [15]. Recently, a structure zone model for sculptured silver thin films that were deposited at low substrate temperatures was investigated. A $T_{\mathrm{s}} / T_{\mathrm{m}}$ ratio of less than 0.1 or in the range $0.2<T_{\mathrm{s}} / T_{\mathrm{m}}<0.3$ yields a columnar structure [16]. However, the growth of metal helices depends on particular values of the coating parameters, including the substrate temperature, the spin rate, the deposition rate, the deposition angle, and the material species [2]. According to works on the growth of plasmonic nanohelices using GLAD, an optimum spiral silver nanohelix array was obtained by matching the substrate spin rate with the deposition rate and maintaining the substrate temperature at $-140^{\circ} \mathrm{C}$. Systematic investigations of the influence of coating parameters on the nanohelical structure are still lacking.

In this work, nanohelices were deposited on BK7 glass substrates in an electron evaporation system; during this process, the substrate was spun at a spin rate of $\omega$, and its normal was tilted at a deposition angle of $\theta$ from the direction of incidence of the vapor. The center of the substrate and the evaporation source were vertically separated by $290 \mathrm{~mm}$. The substrate cooling was achieved by introducing liquid nitrogen or iced water passing through a stainless steel pipe underneath the substrate holder. The holder was made of copper and was 10-mm-thick. The thickness of the Ag nanohelix array was controlled using a crystal oscillator during deposition. A background pressure of $4 \times 10^{-6}$ torr was achieved by pumping before evaporation. A K-type thermocouple was directly mounted next to the substrate on the holder surface. The variations in the morphology of the Ag nanohelices with the deposition rate, the substrate temperature, the spin rate, and the deposition angle were investigated.

\section{Experimental Methods}

\subsection{Nanohelices Grown at Different Deposition Rates and Substrate Spin Rates}

Three one-turn silver nanohelix arrays were deposited at deposition rates of $0.1 \mathrm{~nm} / \mathrm{s}, 0.3 \mathrm{~nm} / \mathrm{s}$, and $0.6 \mathrm{~nm} / \mathrm{s}$. The deposition angle was fixed at $86^{\circ}$. The spin rate was fixed at $\omega=0.018 \mathrm{rpm}$. The substrate was cooled with ice water to a fixed temperature of $10^{\circ} \mathrm{C}$. Figure 1 shows top-view and cross-sectional SEM images of the morphology of each sample. The morphology is described in terms of morphological parameters of mean pitch length $(p)$, wire diameter $(d)$, and radius of curvature $(R)$. About the morphology measurement, the software Image [17] was applied to analyze the SEM images. The contract value is set at 10, other parameters including brightness and saturation values are set at their initial values of 0 and 1, respectively. The morphology parameters are measured by analyzing the SEM images shown in this paper. The pitch length and wire diameter are taken from the cross-sectional image of nanohelices. The radius of curvature and density are taken from the top-view of nanohelices. These parameters are defined as in the inset in Figure 1. The number density of each sample $(n)$ is defined as the mean number of nanohelices per $\mu \mathrm{m}^{2}$ on the substrate surface. The structure of sample-v1 is similar to an upright nanocolumnar structure because the substrate spin rate was too fast to grow nanohelices at the deposition rate of $0.1 \mathrm{~nm} / \mathrm{s}$. The samples that were deposited at $0.3 \mathrm{~nm} / \mathrm{s}$ (sample-v2) and $0.6 \mathrm{~nm} / \mathrm{s}$ (sample-v3) had helical structures. The structure of sample-v2, deposited at $0.3 \mathrm{~nm} / \mathrm{s}$, comprised clearer and more discrete nanohelices than sample-v3. The optimum morphology parameters are $(p, d, R)=(200 \mathrm{~nm}, 71.4 \mathrm{~nm}, 115 \mathrm{~nm})$. The top-view of sample-v3 reveals that the helices are densely packed and overlap each other.

Subsequently, the deposition rate was fixed at $0.3 \mathrm{~nm} / \mathrm{s}$ and the spin rate was tuned from 0.012 to $0.035 \mathrm{rpm}$ to fabricate five nanohelix arrays. The substrate was cooled to $10{ }^{\circ} \mathrm{C}$, and the deposition angle was fixed at $86^{\circ} \mathrm{C}$. Figure 2 shows the top-view and cross-sectional SEM images of the morphologies of samples that were deposited at $\omega=0.012 \mathrm{rpm}$ (sample- $\omega 1), \omega=0.018 \mathrm{rpm}$ (sample- $\omega 2), \omega=0.023 \mathrm{rpm}$ (sample- $\omega 3), \omega=0.029 \mathrm{rpm}$ (sample- $\omega 4$ ), and $\omega=0.035 \mathrm{rpm}$ (sample- $\omega 5$ ). 
Table 1 lists the morphological parameters and number density of each sample. The helical structure did not grow at $\omega=0.035 \mathrm{rpm}$. Clear and spiral-like nanohelices were grown at $\omega=0.018 \mathrm{rpm}$ and $\omega=0.023 \mathrm{rpm}$. Sample- $\omega 2$ has morphological parameters of $(p, d, R)=(200 \mathrm{~nm}, 71.4 \mathrm{~nm}, 115 \mathrm{~nm})$. Sample-w3 has morphological parameters of $(p, d, R)=(188.6 \mathrm{~nm}, 57.1 \mathrm{~nm}, 88.6 \mathrm{~nm})$. The pitch length, wire diameter, and radius of curvature decrease as the spin rate increases. The pitch angle and number density increase with the spin rate.

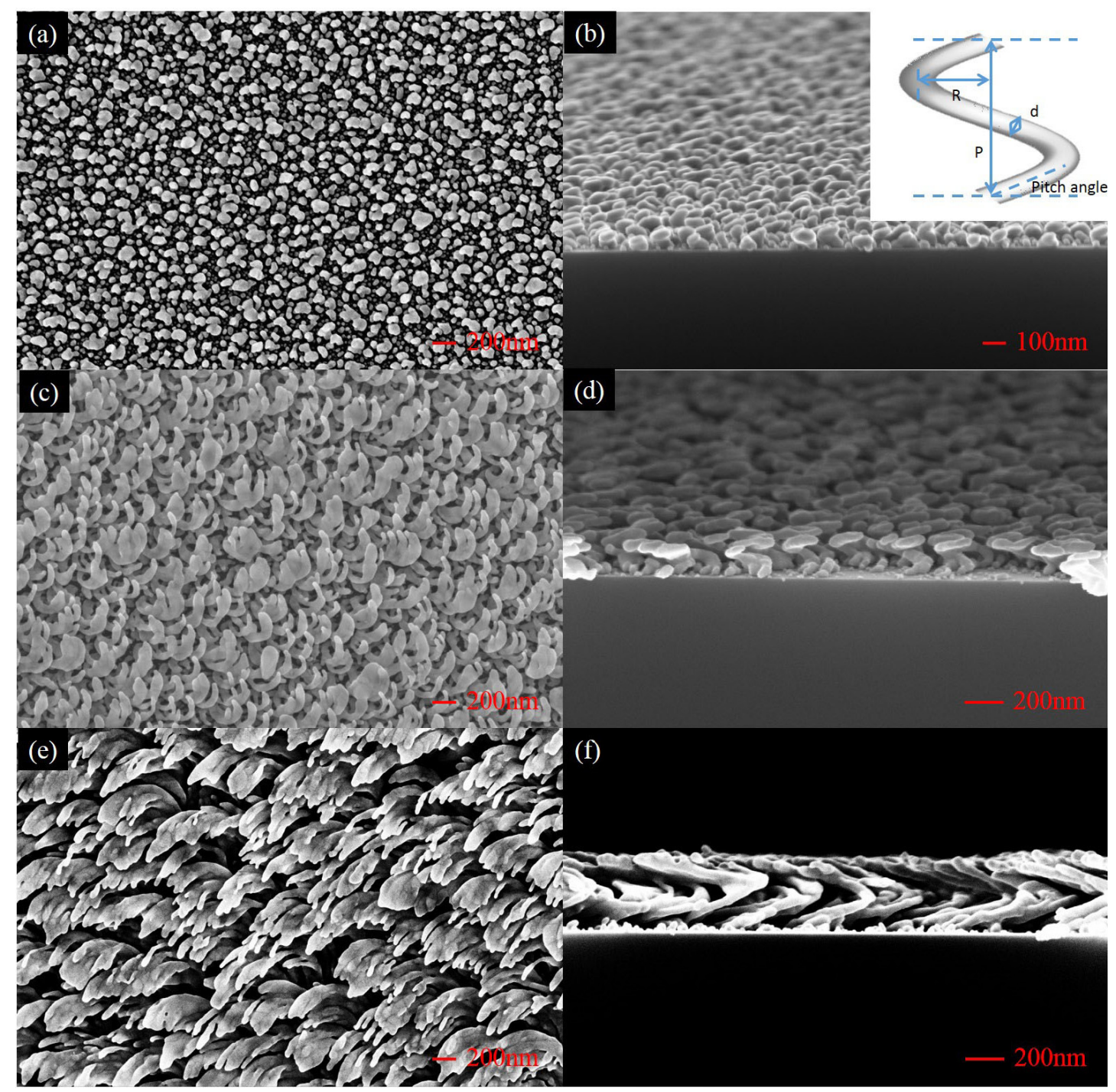

Figure 1. Top-view and cross-section SEM images of Ag nanohelix arrays deposited at deposition rates of $(\mathbf{a}, \mathbf{b}) 0.1 \mathrm{~nm} / \mathrm{s},(\mathbf{c}, \mathbf{d}) 0.3 \mathrm{~nm} / \mathrm{s}$ and $(\mathbf{e}, \mathbf{f}) 0.6 \mathrm{~nm} / \mathrm{s}$.

Table 1. Pitch length $(p)$, wire diameter $(d)$, radius of curvature $(R)$, and density $(n)$ of sample- $\omega j$, $j=1,2,3,4,5$.

\begin{tabular}{|c|c|c|c|c|}
\hline Sample- $\omega j$ & $\begin{array}{l}\text { Pitch Length } p \\
\quad(\mathrm{~nm})\end{array}$ & $\begin{array}{l}\text { Wire Diameter } d \\
(\mathrm{~nm})\end{array}$ & $\begin{array}{c}\text { Radius of Curvature } R \\
(\mathrm{~nm})\end{array}$ & $\begin{array}{c}\text { Density } n \\
\left(1 / \mu \mathrm{m}^{2}\right)\end{array}$ \\
\hline sample- $\omega 1$ & $210 \pm 19$ & $90.5 \pm 10$ & $140.1 \pm 15$ & 15 \\
\hline sample- $\omega 2$ & $200 \pm 15$ & $71.4 \pm 8$ & $115 \pm 12$ & 22 \\
\hline sample- $\omega 3$ & $188.6 \pm 14$ & $57.1 \pm 7$ & $88.6 \pm 12$ & 27 \\
\hline sample- $\omega 4$ & $130.7 \pm 10$ & $45.7 \pm 5$ & $57.1 \pm 8$ & 34 \\
\hline sample- $\omega 5$ & - & - & - & 42 \\
\hline
\end{tabular}




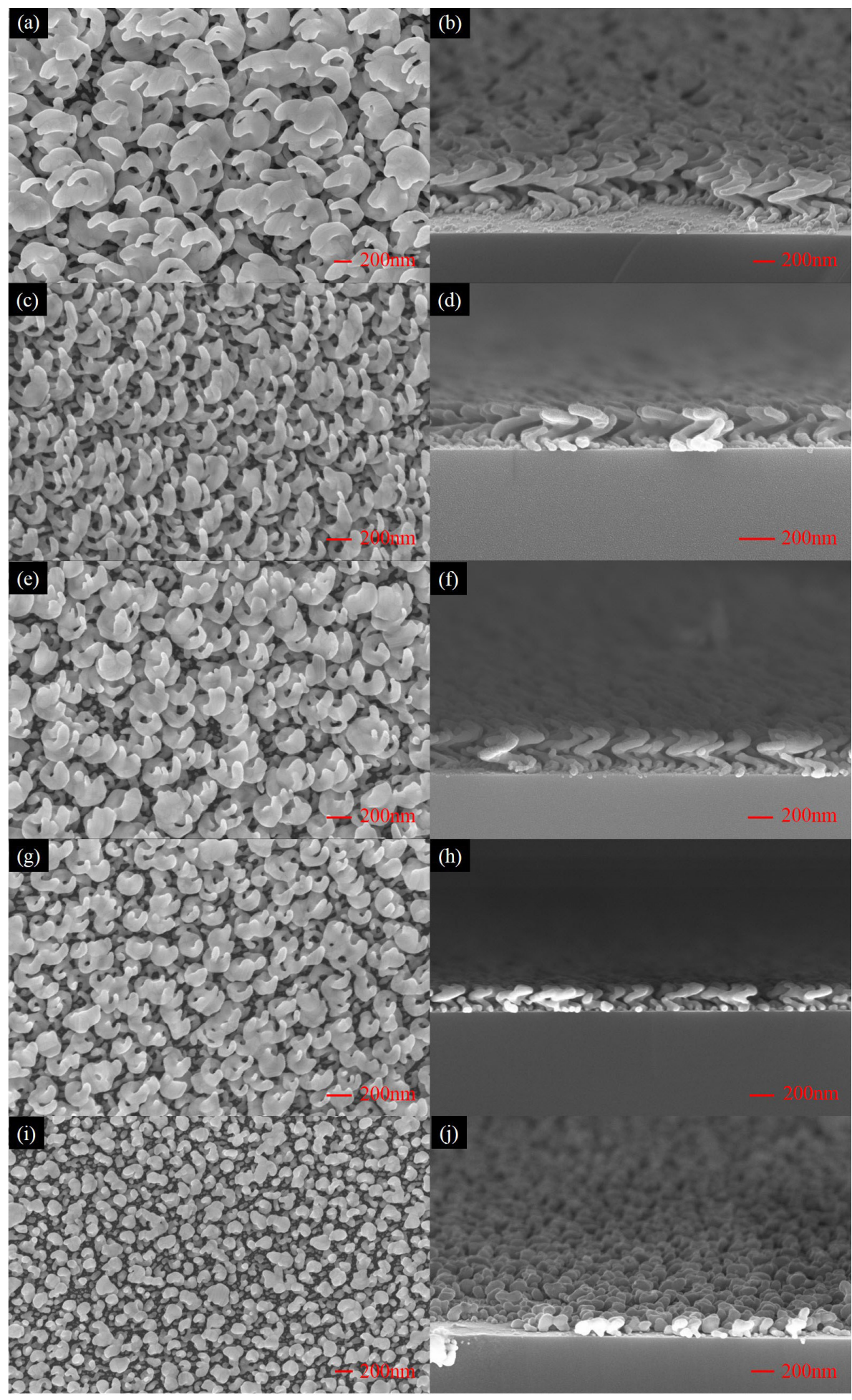

Figure 2. Top-view and cross-section SEM images of Ag nanohelix arrays deposited at spin rates of $(\mathbf{a}, \mathbf{b}) 0.012 \mathrm{rpm},(\mathbf{c}, \mathbf{d}) 0.018 \mathrm{rpm},(\mathbf{e}, \mathbf{f}) 0.023 \mathrm{rpm},(\mathbf{g}, \mathbf{h}) 0.029 \mathrm{rpm}$ and $(\mathbf{i}, \mathbf{j}) 0.035 \mathrm{rpm}$.

\subsection{Nanohelices Grown at Different Deposition Angles and Substrate Temperatures}

Four nanohelix arrays were deposited by tuning the deposition angles under the condition that the deposition rate and substrate spin rate were fixed at $v=0.3 \mathrm{~nm} / \mathrm{s}$ and $\omega=0.018 \mathrm{rpm}$, respectively. The deposition angle was varied from $\theta=80^{\circ}$ to $\theta=89^{\circ}$ to fabricate four nanohelix arrays. The substrate temperature was cooled to $10^{\circ} \mathrm{C}$. Figure 3 shows top-view and cross-sectional SEM images of the 
morphologies of samples that were deposited at $\theta=80^{\circ}$ (sample- $\theta 1$ ), $\theta=83^{\circ}$ (sample- $\theta 2$ ), $\theta=86^{\circ}$ (sample- $\theta 3$ ), and $\theta=89^{\circ}$ (sample- $\theta 4$ ). The figure shows that the morphology of nanohelices is very sensitive to the deposition angle. At a deposition angle of $89^{\circ}$, the number density of nanoparticles is low and the helical structure is not obvious. However, the two nanohelix arrays that are deposited at $\theta=83^{\circ}$ and $\theta=86^{\circ}$ are densely packed. Only sample- $\theta 2$ and sample- $\theta 3$ are well-structured. Sample- $\theta 3$ has morphological parameters of $(p, d, R)=(200 \mathrm{~nm}, 71.4 \mathrm{~nm}, 111.5 \mathrm{~nm})$. As the deposition angle declines from $86^{\circ}$ to $83^{\circ}$, the radius of curvature decreases greatly from $111.5 \mathrm{~nm}$ to $60.1 \mathrm{~nm}$, and the number density increases from 22 to 75 . The aforementioned quantities including pitch length, wire diameter, radius of curvature, and density of four samples are listed in Table 2.

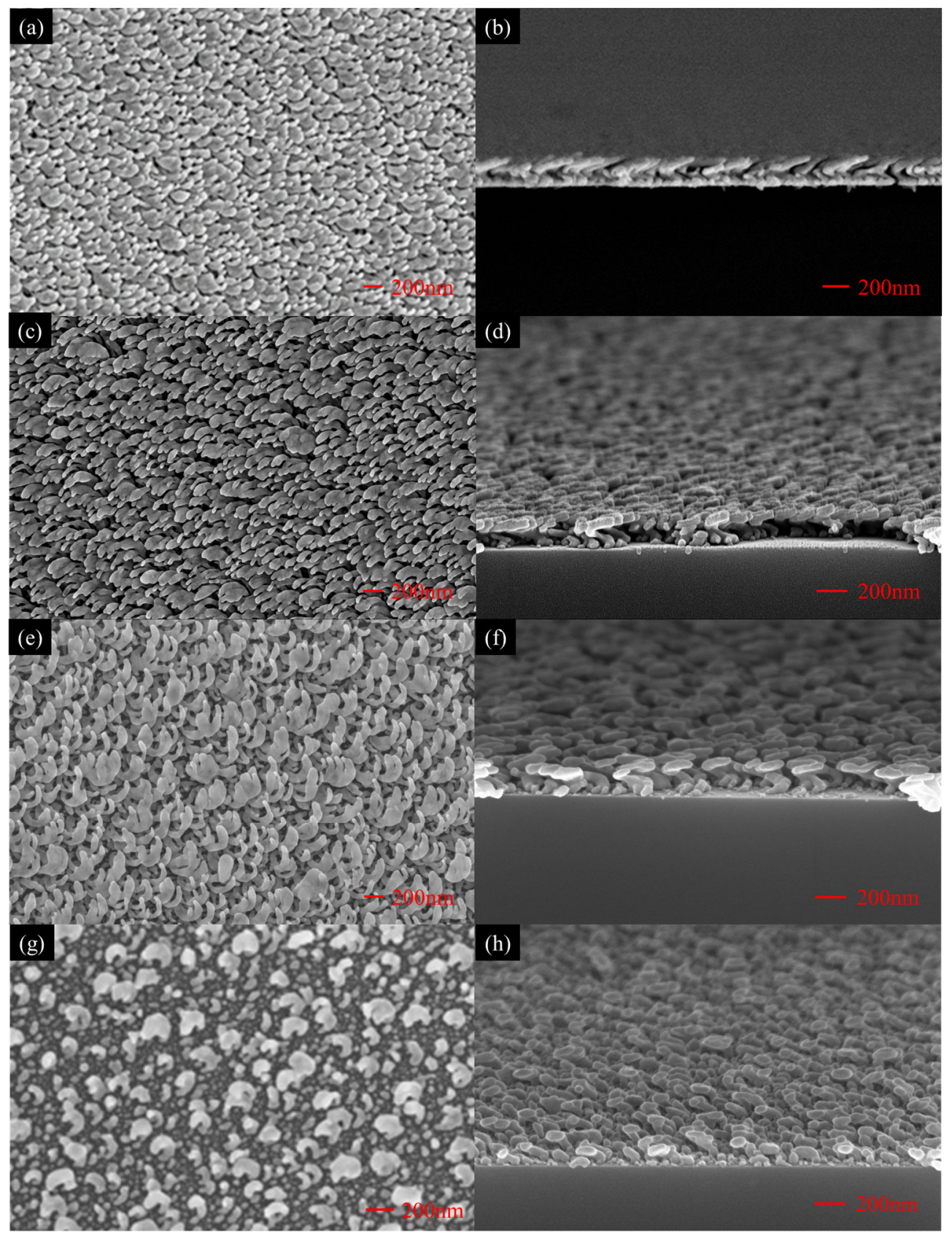

Figure 3. Top-view and cross-section SEM images of Ag nanohelix arrays deposited at deposition angles of $(\mathbf{a}, \mathbf{b}) 80^{\circ} ;(\mathbf{c}, \mathbf{d}) 83^{\circ} ;(\mathbf{e}, \mathbf{f}) 86^{\circ}$ and $(\mathbf{g}, \mathbf{h}) 89^{\circ}$. 
Table 2. Pitch length $(p)$, wire diameter $(d)$, radius of curvature $(R)$, and density $(n)$ of sample- $\theta j$, $j=1,2,3,4$.

\begin{tabular}{|c|c|c|c|c|}
\hline Sample- $\theta j$ & $\begin{array}{l}\text { Pitch Length } p \\
\text { (nm) }\end{array}$ & $\begin{array}{l}\text { Wire Diameter } d \\
(\mathrm{~nm})\end{array}$ & $\begin{array}{c}\text { Radius of Curvature } R \\
(\mathbf{n m})\end{array}$ & $\begin{array}{c}\text { Density } n \\
\left(1 / \mu \mathrm{m}^{2}\right)\end{array}$ \\
\hline sample- $\theta 1$ & $167.3 \pm 12$ & $79.4 \pm 8$ & $50.3 \pm 5$ & 82 \\
\hline sample- $\theta 2$ & $145.4 \pm 11$ & $74.6 \pm 8$ & $60.1 \pm 5$ & 75 \\
\hline sample- $\theta 3$ & $200 \pm 15$ & $71.4 \pm 8$ & $111.5 \pm 12$ & 22 \\
\hline sample- $\theta 4$ & $83.5 \pm 17$ & $58.8 \pm 10$ & $54.1 \pm 13$ & 11 \\
\hline
\end{tabular}

Deposition was carried out at two substrate temperatures. The deposition rate and substrate spin rate were fixed at $v=0.3 \mathrm{~nm} / \mathrm{s}$ and $\omega=0.018 \mathrm{rpm}$, respectively. The deposition angle was fixed at $86^{\circ}$. In our experiment, liquid nitrogen and iced water were used to cool the substrate. The substrate cooling was achieved by introducing liquid nitrogen or iced water through a stainless steel pipe underneath the substrate holder. The deposition started after the deposition rate and the substrate temperature became stable. Therefore, the controlled temperatures were restricted to $-140{ }^{\circ} \mathrm{C}$ and $10^{\circ} \mathrm{C}$. Figure 4 shows top-view and cross-sectional SEM images of morphologies of the samples that were deposited at $T=-140{ }^{\circ} \mathrm{C}$ (sample-T1). Figure 1 shows the morphology of the sample that was deposited at $T=10^{\circ} \mathrm{C}$ (sample-T2). Table 3 presents the difference between the morphologies of two samples. The wire diameter of the nanohelices that are deposited at $T=-140{ }^{\circ} \mathrm{C}$ is only $39.2 \mathrm{~nm}$, whereas that of the nanohelices that are deposited at $T=10^{\circ} \mathrm{C}$ is $71.4 \mathrm{~nm}$. Sample-T1 has a radius of curvature of $128.5 \mathrm{~nm}$ and a number density of 51, which exceed those of sample-T2.

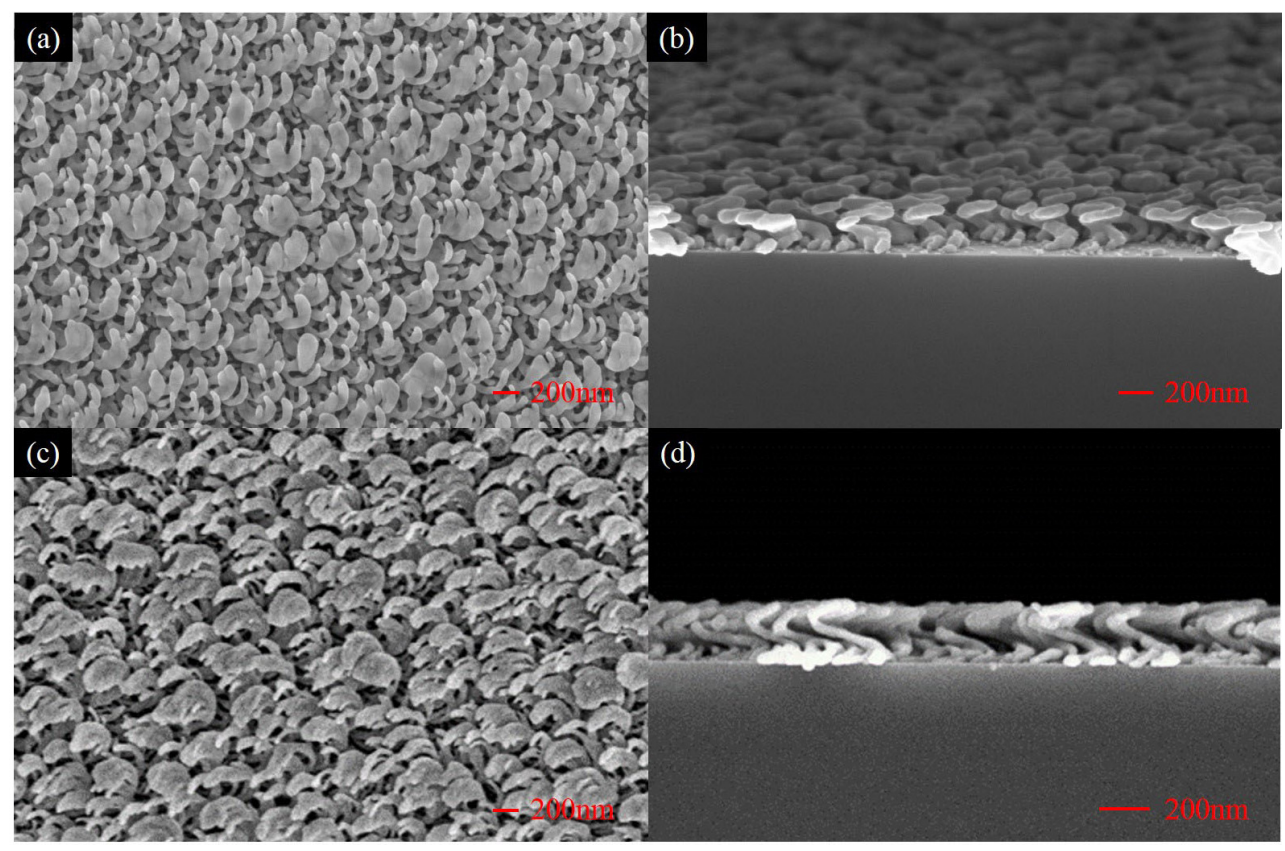

Figure 4. Top-view and cross-section SEM images of Ag nanohelix arrays deposited at substrate temperatures of $(\mathbf{a}, \mathbf{b}) 10^{\circ} \mathrm{C}$ and $(\mathbf{c}, \mathbf{d})-140^{\circ} \mathrm{C}$.

Table 3. Pitch length $(p)$, wire diameter $(d)$, radius of curvature $(R)$, and density $(n)$ of sample-Tj, $j=1,2$.

\begin{tabular}{|c|c|c|c|c|}
\hline Sample-T $j$ & $\begin{array}{l}\text { Pitch Length } p \\
\quad(\mathrm{~nm})\end{array}$ & $\begin{array}{l}\text { Wire Diameter } d \\
(\mathrm{~nm})\end{array}$ & $\begin{array}{c}\text { Radius of Curvature } R \\
(\mathrm{~nm})\end{array}$ & $\begin{array}{c}\text { Density } n \\
\left(1 / \mu \mathrm{m}^{2}\right)\end{array}$ \\
\hline sample-T1 & $197 \pm 13$ & $39.2 \pm 3$ & $128.5 \pm 10$ & 51 \\
\hline sample-T2 & $200 \pm 15$ & $71.4 \pm 8$ & $111.5 \pm 12$ & 22 \\
\hline
\end{tabular}




\section{3. g-Factor of Differently Sized Nanohelix Arrays}

Since nanohelix is a typical chiral structure, the circular polarization-dependent plasmonic effect is an important optical property. By illuminating the nanohelices with different circular polarized light, the difference of extinctance presents the chiral localized plasmonic effect that is critical in chiral molecule detection. In this work, the $g$-factor $(g)$ quantifies circular dichroism and is defined as the equation,

$$
g=\left(\mathrm{E}_{\mathrm{r}}-\mathrm{E}_{1}\right) /\left(\mathrm{E}_{\mathrm{r}}+\mathrm{E}_{1}\right)
$$

where the extinctance $E_{\mathrm{r}}\left(E_{1}\right)$ is measured by illuminating the sample with right-handed (left-handed) circular polarized light. The measurement of circular dichroism applied two linear polarizers (Newport 5524, Irvine, CA, USA) and an achromatic waveplate (ThorLabs AQWP05M-600, Newton, NJ, USA) in front of the monochromator (JobinYvon iHR320, Kyoto, Japan) with a halogen lamp source (Newport 66882, Irvine, CA, USA) to generate circular polarized waves over a wavelength ranging from 400 to $700 \mathrm{~nm}$. The signals were collected by a photomultiplier (Hamamatsu R636-10, Shizuoka, Japan). The transmittance and reflectance spectra associated with right-handed and left-handed incident light were measured to derive extinctance spectra.

The $g$-factor spectra of the samples that were deposited at different spin rates were measured. As shown in Figure 5, the $g$-factor peak is blue-shifted from 659 to $400 \mathrm{~nm}$ as the spin rate increases from 0.012 to $0.029 \mathrm{rpm}$ owing to the shrinking of the nanohelices. The wavelength at which circular dichroism occurs is proportional to the pitch length and radius of the curvature of the helix. The radius of curvature decreases from 140.1 to $57.1 \mathrm{~nm}$ as the spin rate increases from 0.012 to $0.029 \mathrm{rpm}$.

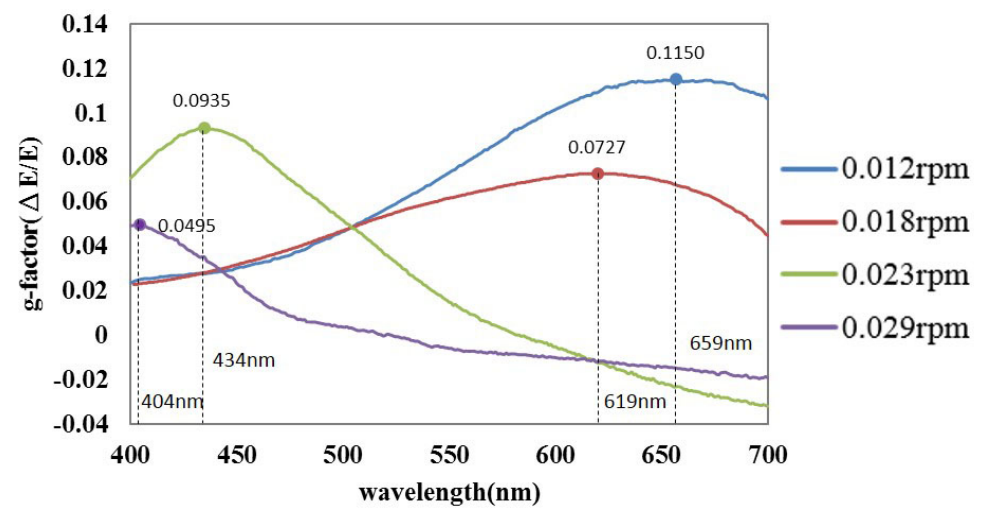

Figure 5. $g$-Factor spectra of samples deposited at $0.012 \mathrm{rpm}, 0.018 \mathrm{rpm}, 0.023 \mathrm{rpm}$, and $0.029 \mathrm{rpm}$.

\section{Discussion and Conclusions}

In conclusion, various silver nanohelices were formed by tuning the coating parameters. The average arm width of the silver nanohelices that were deposited at $10{ }^{\circ} \mathrm{C}$ was thicker than that of the nanohelices that were deposited at $-140^{\circ} \mathrm{C}$. According to the zone model [16], the overlap between adjacent nanohelices was improved by increasing the substrate temperature. The substrate cooling with liquid nitrogen would cause the slanted deposited rods to become densely distributed. Compared with the aforementioned case, the substrate cooling with ice water would cause the slanted rods to become distributed more sparsely, in turn causing larger spacing between rods to reduce the overlap effect between adjacent rods. The morphology of the nanohelix array is highly sensitive to the deposition angle. The morphology of the nanohelices can be controlled by changing the substrate spin rate and deposition rate. Measurements reveal that the circular dichroism is proportional to the radius of curvature. The maximum g-factor at blue, green, and red wavelengths can be obtained by varying the size of the nanohelices. To ensure that the circular dichroism occurs at the desired wavelengths, a nanohelix array can be produced by setting suitable deposition conditions as determined by the analysis herein. 
Acknowledgments: The authors would like to thank the Ministry of Science and Technology of the Republic of China, Taiwan, for financially supporting this research under Contract No. MOST 105-2221-E-027-072-MY3.

Author Contributions: Yi-Jun Jen conceived the idea and supervised the whole work. Yueh Weng Lin, Yu-Jie Huang, and Teh-Li Chan deposited the films and performed measurements. Yi-Jun Jen, Yueh Weng Lin, and Yu-Jie Huang analyzed the data. Yi-Jun Jen wrote the article. All authors meet the three criteria for authorship.

Conflicts of Interest: The authors declare no conflict of interest.

\section{References}

1. Gansel, J.K.; Thiel, M.; Rill, M.S.; Decker, M.; Bade, K.; Saile, V.; Freymann, G.V.; Linden, S.; Wegener, M. Gold helix photonic metamaterial as broadband circular polarizer. Science 2009, 325, 1513-1515. [CrossRef] [PubMed]

2. Gansel, J.K.; Wegener, M.; Burger, S.; Linden, S. Gold helix photonic metamaterials: A numerical parameter study. Opt. Express 2010, 18, 1059-1069. [CrossRef] [PubMed]

3. Huang, Z.; Bai, F. Wafer-scale, three-dimensional helical porous thin films deposited at a glancing angle. Nanoscale 2014, 6, 9401-9409. [CrossRef] [PubMed]

4. Steele, J.J.; Brett, M.J. Nanostructure engineering in porous columnar thin films: Recent advances. J. Mater. Sci. Mater. Electron. 2007, 18, 367-379. [CrossRef]

5. Mark, A.G.; Gibbs, J.G.; Lee, T.C.; Fischer, P. Hybrid nanocolloids with programmed three-dimensional shape and material composition. Nat. Mater. 2013, 12, 802-807. [CrossRef] [PubMed]

6. Jensen, M.O.; Brett, M.J. Periodically structured glancing angle deposition thin films. IEEE Trans. Nanotechnol. 2005, 4, 269-277. [CrossRef]

7. Summers, M.A.; Brett, M.J. Optimization of periodic column growth in glancing angle deposition for photonic crystal fabrication. Nanotechnology 2008, 19, 415203. [CrossRef] [PubMed]

8. Deng, J.; Huang, Z. Radiative loss-determined circular dichroism of plasmonic nanospirals with bendable stability of chiroptical activity. RSC Adv. 2016, 6, 84348-84352. [CrossRef]

9. Jen, Y.J.; Huang, Y.J.; Liu, W.C.; Lin, Y.W. Densely packed aluminum-silver nanohelices as an ultra-thin perfect light absorber. Sci. Rep. 2017, 7, 39791. [CrossRef] [PubMed]

10. Jen, Y.J.; Huang, J.W.; Liu, W.C.; Chan, S.; Tseng, C.H. Glancing angle deposited gold nanohelix arrays on smooth glass as three-dimensional SERS substrates. Opt. Mater. Express 2016, 6, 697-704. [CrossRef]

11. Jen, Y.J.; Chan, S.; Huang, J.W.; Jheng, C.Y.; Liu, W.C. Self-shadowing deposited pure metal nanohelix arrays and SERS application. Nanoscale Res. Lett. 2015, 10, 498. [CrossRef] [PubMed]

12. Bai, F.; Deng, J.; Yang, M.; Fu, J.; Ng, J.; Huang, Z. Two chiroptical modes of silver nanospirals. Nanotechnology 2016, 27, 115703. [CrossRef] [PubMed]

13. Bai, F.; Lau, W.F.; Yang, L.; Roy, V.A.L.; Huang, Z.F. Dramatic enhancement of enantiomer differentiation induced by chiral nanoplasmons. Sci. Adv. Today 2016, 2, 25250.

14. Lau, W.; Yang, L.; Bai, F.; Huang, Z. Weakening circular dichroism of plasmonic nanospirals induced by surface grafting with alkyl ligands. Small 2016, 12, 6698-6702. [CrossRef] [PubMed]

15. Thornton, J.A. Influence of apparatus geometry and deposition conditions on the structure and topography of thick sputtered coatings. J. Vac. Sci. Technol. 1974, 11, 666. [CrossRef]

16. Singh, D.P.; Goel, P.; Singh, J.P. Revisiting the structure zone model for sculptured silver thin films deposited at low substrate temperatures. J. Appl. Phys. 2012, 112, 104324. [CrossRef]

17. Image Processing Software: ImageJ, National Institutes of Health, USA, Version 1.48s. Available online: https:/ /imagej.nih.gov/ij (accessed on 27 August 2017).

(C) 2017 by the authors. Licensee MDPI, Basel, Switzerland. This article is an open access article distributed under the terms and conditions of the Creative Commons Attribution (CC BY) license (http:/ / creativecommons.org/licenses/by/4.0/). 